\title{
Synthesis of 4- (Thieno [3, 2-d] pyrimidin-4-yl) Morpholine Huajun Lei ${ }^{1}$, Linxiao Wang ${ }^{1}$, Yingjia Xiong ${ }^{1}$, and Zhou Lan ${ }^{1, a}$ \\ ${ }^{1}$ School of Pharmacy, Jiangxi Science \& Technology Normal University, Nanchang 330013, China \\ *alan8709@yeah.net
}

Keywords: 4-(Thieno[3,2-d]pyrimidin-4-yl)morpholine; Synthesis; Intermediate

\begin{abstract}
Thieno[3,2-d]pyrimidin-4-yl)morpholine derivatives is an important intermediate which inhibited tumor necrosis factor alpha and nitric oxide. In this work, a rapid and green synthetic method for compound (5) was established. The compound (5) was synthesized from the commercially available methyl 3-aminothiophene-2-carboxylate and formamidine acetate through three steps including condensation reaction, chlorination, and nucleophilic substitution. The structure was confirmed by ${ }^{1} \mathrm{H}$ NMR and MS. Furthermore, the total yield of the three steps was $43 \%$.
\end{abstract}

\section{Introduction}

Thienopyrimidine is a key intermediate which has many applications in research work. Many articles have reported that a series of substituted thienopyrimidine derivatives were synthesized[1-2]. Most of the thienopyrimidine derivatives inhibited tumor necrosis factor alpha and nitric oxide, it was also confirmed to be a potent inhibitor for acute myeloid leukemia. In addition, the research team occasionally find that the thienopyrimidine analogues didn't IKKb inhibitory activities, nevertheless it's has a effective on acute myeloid leukemia[3]. At the same times, many articles have repoted that thienopyrimidine derivatives have a antimicrobial activity[4-6]. One of the literature points out some 2-substituted thienopirimidin-4-one derivatives may serve as novel spasmolytic agents to treat disorders of smooth muscle function. In addition to, thieno[3,2d]pyrimidine derivatives inhibit PI3 kinase p110 $\alpha$. Phosphatidylinositol-3-kinase (PI3K) is an important cancer target which deregulates the PI3K/Akt signaling pathway in a wide variety of tumors[7]. The representative of the PI3K inhibitor is GDC-0941, and it's structure in Fig 1. The latest literature shows that thieno[3,4-d]-pyrimidine as a common heterocyclic nucleus, the research team reinstall this functionality into a new isomorphic RNA alphabet, with higher structural and electronic similarity to the native purines, therefore it can be better simulate the role of purine[8]. While the thieno[3,4-d]-pyrimidine has a highly emissive and valuable as illustrated by several applications[9] and its structure was shown in Fig 1. At the same times, some literatures have reported that the thienopyrimidine core can be used in preparing potent P2Y12 inhibitors[10]. Most of the synthetic methods of thieno[3,2-d]-pyrimidine which was reported in the literature have the drawbacks such as low yield, harmful to environment and long synthetic route. Based on our previous research, through three steps we have synthesized the title compound of thienopyrimidine, including condensation reaction, chlorination, and nucleophilic substitution, and those methods easy, non-toxic, and green.

In this study, we designed and optimized the synthetic methods for 4-(thieno[3,2-d]pyrimidin4-yl)morpholine (5) and made it more suitable for industrial production. The target compound structure was shown in Fig 1. 
<smiles>CS(=O)(=O)N1CCN(Cc2cc3nc(-c4cccc5[nH]ncc45)nc(N4CCOCC4)c3s2)CC1</smiles>

GDC-0941<smiles>[R]c1scc2cncnc12</smiles>

Thieno[3,4- $d]$-pyrimidin<smiles>c1nc(N2CCOCC2)c2sccc2n1</smiles>

Target compound

Figure 1. Sructures of GDC-0941, thieno[3,4- $d]$-pyrimidine and target compound

\section{Materials and Methods}

All chemicals and reagents used in current study were of analytical grade. NMR spectra were performed using Bruker $300 \mathrm{MHz}$ spectrometers (Bruker Bioscience, Billerica, MA, USA) with TMS as an internal standard. Mass spectra (MS) were taken in ESI mode on Agilent 1100 LC-MS (Agilent, Palo Alto, CA, USA). Elemental analysis was determined on a Carlo-Erba 1106 Elemental analysis instrument (Carlo Erba, Milan, Italy). All the materials were obtained from commercial suppliers and used without purification, unless otherwise specified. Yields were not optimized. TLC analysis was carried out on silica gel plates GF254 (Qindao Haiyang Chemical, China).

\section{Synthesis of Compounds}

The structures and the synthetic route were shown in Scheme 1.

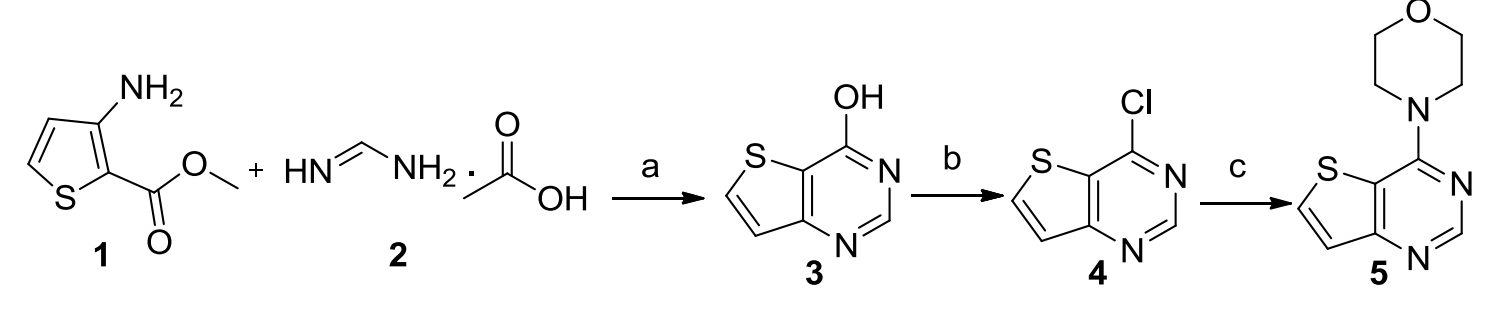

Scheme 1. The synthetic route of compound (5)

Reagents and Conditions: (a) ethanol, $80^{\circ} \mathrm{C}, 14 \mathrm{~h}$. (b) phosphoryl trichloride, DMF, $80^{\circ} \mathrm{C}, 3 \mathrm{~h}$. (c) morpholine, ethanol, $78^{\circ} \mathrm{C}, 5 \mathrm{~h}$.

Thieno[3,2-d]Pyrimidin-4-Ol (3). Methyl 3-aminothiophene-2-carboxylate (1) (25 g, 160 mmol) and formamidine acetate (2) $(31 \mathrm{~g}, 210 \mathrm{mmol})$ were heated in ethanol $(45 \mathrm{~mL})$ at $80{ }^{\circ} \mathrm{C}$ to give Thieno[3,2- $d$ ] pyrimidin-4-ol (3). After the reaction completed, it was stirred in cold water for one hour, and then the solid precipitate was filtered, washed with cold water. Yield: $(54 \%) .{ }^{1} \mathrm{H}$ NMR (400 MHz, DMSO) $\delta 12.41(\mathrm{~s}, 1 \mathrm{H}), 8.05(\mathrm{~s}, 1 \mathrm{H}), 7.50(\mathrm{~d}, J=0.9 \mathrm{~Hz}, 1 \mathrm{H}), 7.32$ (dd, $J=5.8,1.4 \mathrm{~Hz}$, 1H). MS (ESI): m/z 153.0[M+H] ${ }^{+}$.

4-Chlorothieno[3,2-d]pyrimidine (4). Thieno[3,2-d]pyrimidin-4-ol (3) (3 g, $19.8 \mathrm{mmol}$ ) was suspended in DMF $(15 \mathrm{~mL})$, then phosphoryl trichloride $(35 \mathrm{~mL})$ was added and the mixture stirred at the $80{ }^{\circ} \mathrm{C}$ for $3 \mathrm{~h}$. The reaction precedure was monitored by the TLC, after the reaction completed, it was slowly poured it into ice-cold water, and the mixture was stirred at room temperature. The solid product formed was filtered, washed with water, dried and crystallized from ethanol. Yield 85\%, mp: 115-118 ${ }^{\circ} \mathrm{C} .{ }^{1} \mathrm{H}$ NMR (400 MHz, DMSO) $\delta 8.75(\mathrm{~s}, 1 \mathrm{H}), 8.24$ (d, $\left.J=6.0 \mathrm{~Hz}, 1 \mathrm{H}\right), 7.48$ (d, $J=6.0 \mathrm{~Hz}, 1 \mathrm{H}) . \mathrm{MS}(\mathrm{ESI}): \mathrm{m} / \mathrm{z} 171.9\left[\mathrm{M}+\mathrm{H}^{+}{ }^{+}\right.$. MS (ESI): m/z 153.0[M+H] ${ }^{+}$. 
4-(Thieno[3,2-d]pyrimidin-4-yl)morpholine (5). 4-Chlorothieno[3,2- $d]$ pyrimidine (4) (1.2 g, $6.1 \mathrm{mmol})$ and morpholine $(0.85 \mathrm{~mL})$ were dissolved in ethanol $(15 \mathrm{~mL})$ and refluxed to give the desired compound (5). After the reaction completed, the product was extracted with dichloromethane(DCM) and water, the extract was washed successively with water, dried over anhydrous $\mathrm{Na}_{2} \mathrm{SO}_{4}$, filtered and evaporated, then to give 4-(thieno[3,2- $d$ ] pyrimidin-4-yl)morpholine (5). In 54\% yield, mp: 109-110 ${ }^{\circ} \mathrm{C} .{ }^{1} \mathrm{H}$ NMR (400 MHz, DMSO) $\delta 8.44$ (s, 1H), 7.65 (d, $J=6.6 \mathrm{~Hz}$, $2 \mathrm{H}), 3.91-3.83(\mathrm{t}, 4 \mathrm{H}), 3.78-3.70(\mathrm{t}, 4 \mathrm{H}) . \mathrm{MS}(\mathrm{ESI}): \mathrm{m} / \mathrm{z} 222.0[\mathrm{M}+\mathrm{H}]^{+}$.

\section{Conclusions}

In conclusion, the target compound 4-(thieno[3,2-d]pyrimidin-4-yl)morpholine (5) was synthesized starting from the simple derivatives of methyl 3-aminothiophene-2-carboxylate (1) and formamidine acetate (2) through three steps including nucleophilic substitution, chlorination. The synthetic method of compound (5) and the reactions conditions were optimized, the purity of the product is high. All the compounds structure was confirmed by $1 \mathrm{H}$ NMR spectrum and MS.

\section{Acknowledgments}

We gratefully acknowledge the generous support provided by Project supported by Doctoral Scientific Research Foundation of Jiangxi Science \& Technology Normal University and Program of Key Laboratory of Drug Design and Optimization, Jiangxi Science \& Technology Normal University(300098010306) and College Students' Science and Technology Innovation Project of Jiangxi Province..

\section{References}

[1] Hamed A. A.; Zeid I. F.; Elganzoreye H. H.; et al. Synthesis and Structure of Some Thienopyrimidine Derivatives[J] ChemInform., 2008, 7(139): 809-820.

[2] Guillermo A. M.; Joseph R. G.; Jingdong Su.; et al. Synthesis and Cancer Stem Cell-Based Activity of Substituted 5-Morpholino-7H-thieno[3,2-b]pyran-7-ones Designed as Next Generation PI3K Inhibitors[J] J. Med. Chem., 2013, 56(5):1922-1939.

[3] Chulho L.; Jee S. Y. Gyoonhee H. Identification of a thienopyrimidine derivatives target by a kinome and chemical biology approach[J]. Arch. Pharm. Res., 2015, 38:1575-1581.

[4] Hozien Z. A.; Atta F. M.; Hassan K. M.; et al. Synthesis and Application of Some New Thienopyrimidine Derivatives as Antimicrobial Agents[J]. Chem Inform., 1996, 20(26): 37333755.

[5] El-sherbeny M. A.; El-ashmawy M. B.; El-subbagh H. I.; et al. Synthesis, Antimicrobial and Antiviral Evaluation of Certain Thienopyrimidine Derivatives[J]. Eur. J. Med .Chem., 1995, 5(30): 445-449.

[6] Aly H. M.; Saleh, N. M.; Elhady H. A. Design and Synthesis of Some New Thiophene, Thienopyrimidine and Thienothiadiazine Derivatives of Antipyrine as Potential Antimicrobial Agents[J]. Chem Inform., 2011, 9(46): 4566-4572.

[7] Adrian J. F.; Khatereh A.; Wendy K. A.; et al. The Identification of 2-(1H-Indazol-4-yl)-6-(4methanesulfonyl-piperazin-1-ylmethyl)-4-morpholin-4-yl-thieno[3,2- $d$ ]pyrimidine (GDC0941) as a Potent, Selective, Orally Bioavailable Inhibitor of Class I PI3 Kinase for the Treatment of Cancer[J]. J. Med. Chem., 2008, 51 (18): 5522-5532.

[8] Alexander R. R.; Andrea F.; Yitzhak T. Chemical Mutagenesis of an Emissive RNA Alphabet[J]. J. Am. Chem. Soc., 2015, 137: 14602-14605. 
[9] Dongwon S.; Renatus W.S.; Yitzhak T. Emissive RNA Alphabet[J]. J. Am. Chem. Soc., 2011, 133 (38): 14912-1491.

[10] Steven W. K.; Rhonda M. L.; Barbara A. S.; et al. Thienopyrimidine-based P2Y12 platelet aggregation inhibitors[J]. Bioorg. Med. Chem. Let., 2009, 19: 5919-5923. 